\title{
Measurement of the Cosmic-ray Proton Spectrum with the Fermi Large Area Telescope
}

\section{David M. Green*}

University of Maryland: College Park

Goddard Space Flight Center

E-mail: damgreen@umd.edu

\section{Elizabeth A. Hays}

Goddard Space Flight Center

E-mail: elizabeth.a.hays@nasa.gov

\begin{abstract}
We present the measurement of the cosmic-ray proton spectrum between $54 \mathrm{GeV}$ and $9.5 \mathrm{TeV}$ using 7 years of Pass 8 flight data from the Fermi Large Area Telescope (LAT). We developed a dedicated proton event selection with an acceptance of $0.25 \mathrm{~m}^{2} \mathrm{sr}$. Our analysis yields a large dataset with low statistical uncertainty and low residual contamination for a spectral measurement. The systematic errors associated with the acceptance, energy measurement, Geant4 Monte-Carlo simulations are an order of magnitude larger than the statistical uncertainty. The event selection and spectral measurement of the proton analysis create the opportunity for additional proton analyses with the LAT, such as a dedicated proton anisotropy search.
\end{abstract}

35th International Cosmic Ray Conference - ICRC2017

10-20 July, 2017

Bexco, Busan, Korea

${ }^{*}$ Speaker. 


\section{Introduction}

Protons represent the largest fraction of cosmic rays across all energies and the largest portion of events downloaded from the Fermi Large Area Telescope (LAT). The proton event sample covers the entire LAT mission to date (more than six years of data) and extends to greater than $\mathrm{TeV}$ energies. The flux and spectrum of cosmic-ray protons are crucial to understanding galactic cosmicray origins and propagation. The Fermi Large Area Telescope is in a unique position to investigate the spectral shape and break at $300 \mathrm{GeV}$ observed by Pamela and AMS-02 [1, 2]. We present the preliminary cosmic-ray proton analysis for the Fermi Large Area Telescope.

\section{The Large Area Telescope}

The Large Area Telescope (LAT) is a pair conversion $\gamma$-ray telescope designed to measure high energy $\gamma$-rays from $20 \mathrm{MeV}$ to more than $300 \mathrm{GeV}$. The LAT can be used to study more than $\gamma$-rays-its particle detector capability allows the study of protons to energies over $1 \mathrm{TeV}$. The three subsystems of the LAT are the tracker (TKR), calorimeter (CAL), and anti-coincidence detector (ACD). The LAT consists of $4 \times 4$ towers that measure incident direction and energy of incoming cosmic-rays. Each tower contains a TKR and a CAL module. A TKR module is 18 interlaced silicon strip detectors with 16 tungsten foil conversion layers. The TKR has a depth of 0.1 nuclear interaction lengths $\left(\lambda_{i}\right)$ at normal incidence. The TKR's main purpose is to measure the direction of and incoming cosmic ray; in addition the silicon strip detectors can measure time over threshold (ToT) of signal for each plane in the TKR which can be used to measure charge of the incident cosmic ray. Each CAL module consists of $96 \mathrm{CsI}(\mathrm{Tl})$ crystals in a hodoscopic array of 8 layers and has a total depth at normal incidence of $0.5 \lambda_{i}$. The CAL measures the deposited energy of the cosmic ray and its hodoscopic nature allows the imaging of the shower shape for distinguishing between hadronic and leptonic showers. The final subsystem is the ACD, composed of segmented plastic scintillator that tags $>99.97 \%$ of charged particles and covers the TKR and the CAL. The ACD can be used for measuring the charge of the incoming cosmic ray [5].

The Pass 8 Cosmic-Ray Electron studiy provides an important framework for the preliminary proton analysis [6]. The main challenges of a proton spectrum measurement with the LAT include identification of protons from other cosmic-ray species such as electrons, helium, and other heavier ions $(Z>1)$; the poor energy resolution for hadronic showers due to the shallow depth of the CAL; and the systematic uncertainties in the acceptance and energy measurement.

\section{Spectral Analysis}

To measure the cosmic-ray proton spectrum, a dedicated proton event selection was developed to find a sample of well reconstructed protons with low contamination from other cosmic-ray species. The spectrum is unfolded using TUnfold and a Tikhonov regularization with a response matrix estimated from dedicated and calibrated Geant 4 proton Monte-Carlo simulations [7, 8, 9].

\subsection{Event Selection}

While the majority of events downloaded from the Fermi Gamma-ray Space Telescope are protons, we need to be careful in our selection of cosmic-ray protons for this analysis. The Pass 8 
event reconstruction software is designed for gamma-rays but through a careful event selection we can select well reconstructed cosmic-ray protons. The event selection requires that the event has to trigger the LAT on-board and pass the HI-PASS filter which requires an event to deposit at least $20 \mathrm{GeV}$ in the CAL. Next, a track has to be found and must be well reconstructed using the Pass 8 multivariate direction classifier. The effect of these successive cuts provides a starting acceptance of over $1 \mathrm{~m}^{2}$ sr at $1 \mathrm{TeV}$ as shown in Figure 1.

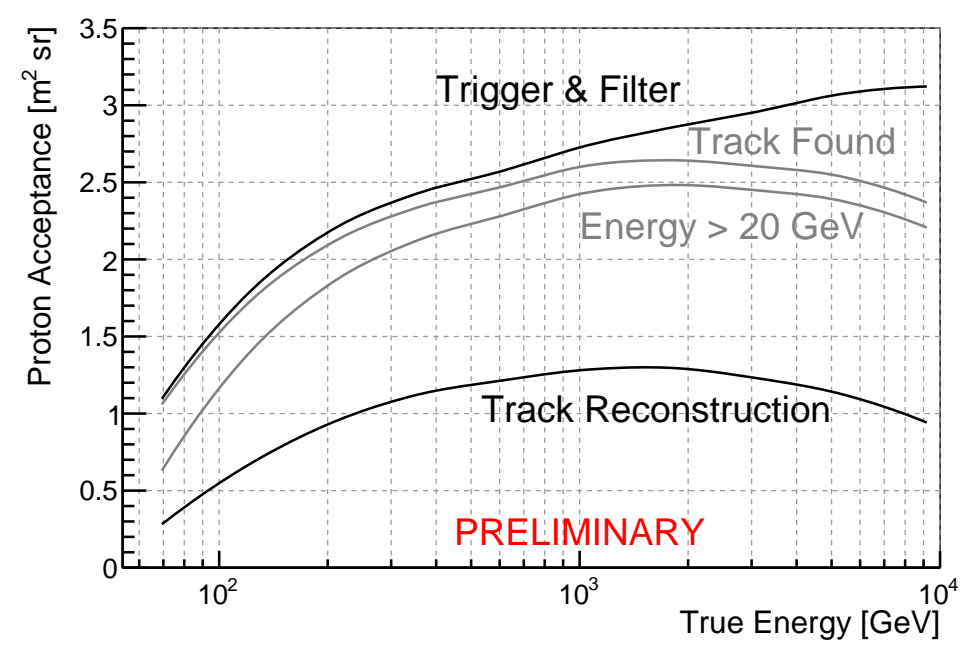

Figure 1: The change in the acceptance from successive cuts from the event selection including trigger, track and energy, minimum distance, direction reconstruction, and energy resolution cuts.

Contamination from other cosmic-ray species, such as helium, carbon, and oxygen, can be upwards of 50\% and need to be efficiently removed from the data sample. Two independent charge measurements from the TKR and the ACD select cosmic-ray protons and reduce residual contamination from $Z>1$ cosmic-ray to less than $1 \%$ as seen in Figure 2. Residual electrons do remain from this event selection but with a contamination less than $10 \%$ and predominately at the lowest energies of this analysis. The residual electron contamination is included as a background and subtracted from the measured event rate before unfolding the spectrum.

A selection which improves energy resolution is applied by selecting events that are well fit by the Pass 8 longitudinal profile energy estimator [10], that do not fall within gaps between CAL modules, and with minimum leakage through the back of the CAL and back into the TKR and which traverses a path length of at least $0.5 \lambda_{i}$ in the CAL. These cuts further reduce the acceptance but improve the energy resolution; the final acceptance for this analysis can be seen in Figure 2 and it's greater than $0.25 \mathrm{~m}^{2} \mathrm{sr}$ at $1 \mathrm{TeV}$. This trade-off is favorable since the proton event rate and acceptance are high enough that there is an estimated 5 million events above $1 \mathrm{TeV}$.

\subsection{Spectral Reconstruction}

The acceptance is calculated from proton Monte-Carlo simulations using equation 3.1 for bin $i$ in true energy. $A_{\mathrm{Gen}}$ and $\Omega_{\mathrm{Gen}}$ are $6 \mathrm{~m}^{2}$ and $4 \pi \mathrm{sr}$ respectively. $N_{\mathrm{Pass}, i}$ is the number of events which pass the entire event selection in bin $i$ and $N_{\mathrm{Gen}, i}$ are the number of events generated in bin $i$. 

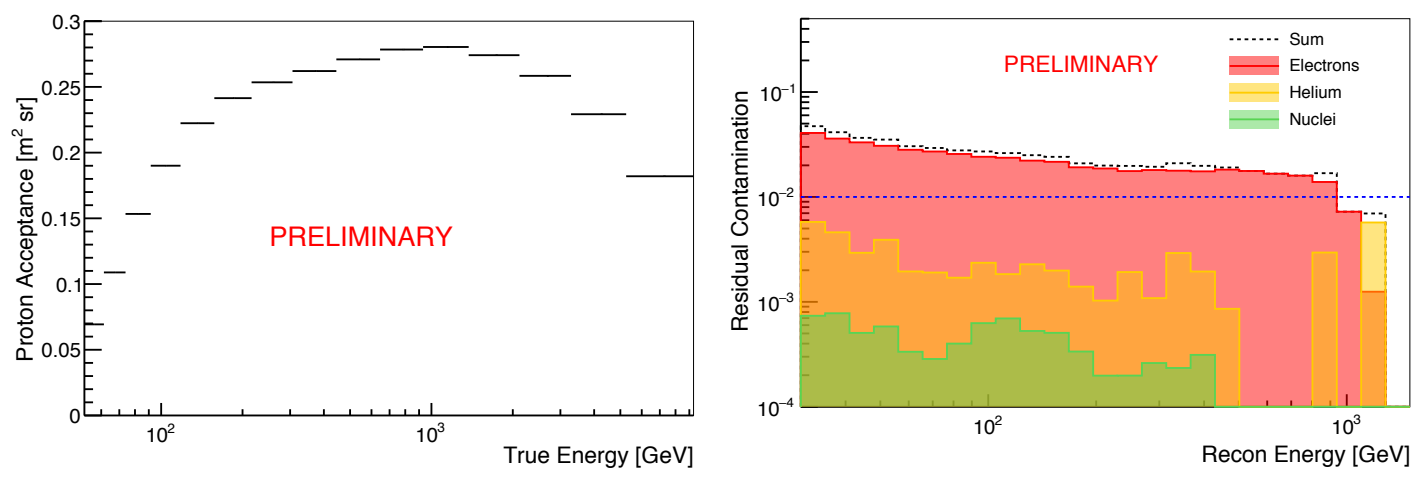

Figure 2: The acceptance of the final event selection (left) in $\mathrm{m}^{2} \mathrm{sr}$ vs true energy in $\mathrm{GeV}$ and the residual contamination (right) from electrons, helium, and $\mathrm{Z}>1$ cosmic-ray nuclei in reconstructed energy in $\mathrm{GeV}$.

$$
A_{i}=A_{\mathrm{Gen}} \times \Omega_{\mathrm{Gen}} \times \frac{N_{\mathrm{Pass}, i}}{N_{\mathrm{Gen}, i}}
$$

To find the cosmic-ray proton spectrum in terms of true energy, the measured event rate is unfolded using the response matrix estimated by dedicated proton Monte-Carlo simulations. The response matrix, shown in Figure 3, is the two dimensional histogram between true energy and reconstructed energy normalized by acceptance in units of $\mathrm{m}^{2} \mathrm{sr}$. The binning on the true energy axis is chosen to be equal to the energy resolution, estimated from dedicated MonteCarlo simulation, with an additional 10\% padding. The padding is included to account for uncertainties associated with the Geant4 proton simulation which may underestimate the energy resolution.

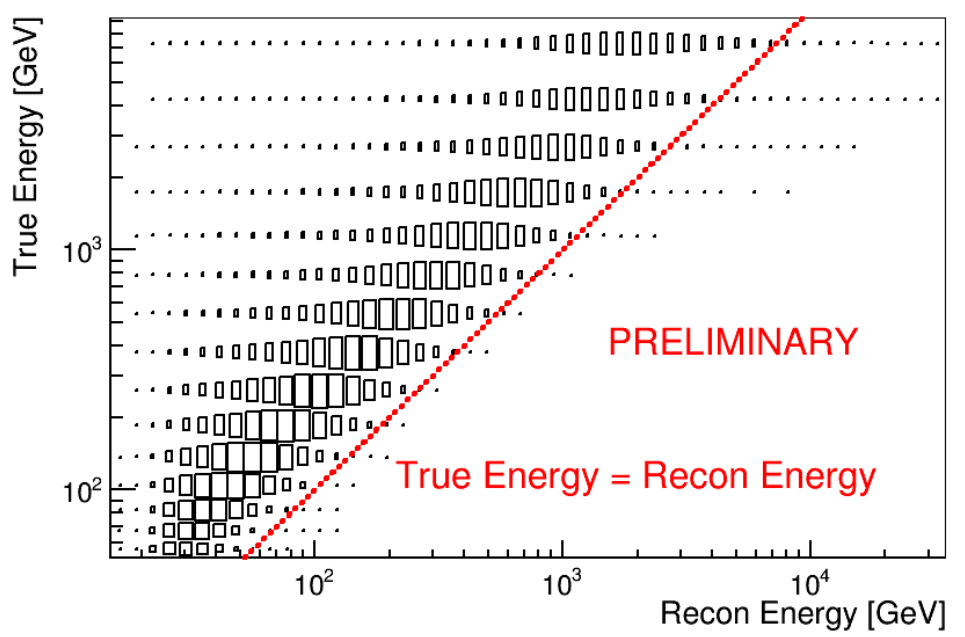

Figure 3: The response of the LAT to protons. This response matrix covers incidence angles up to $70^{\circ}$ off normal incidence. The size of the boxes represent acceptance in units of $\mathrm{m}^{2}$ sr. The response matrix is used to unfold flight data from reconstructed energy to true energy. The reconstructed energy binning is set to 50 bins from $30 \mathrm{GeV}$ to $35 \mathrm{TeV}$ and the true energy binning is determined from the energy resolution. 


$$
J_{E, i}=\frac{N_{i}}{A_{i} \Delta E_{i}}
$$

For the unfolding we use ROOT's TUnfold with a Tikhonov regularization $[11,7,8]$. Equation 3.2 is used to find the differential energy spectrum, divided by the acceptance, shown in figure 2, and the true energy bin width.

\section{Systematic Uncertainties}

Since the acceptance and response matrix are generated from Monte-Carlo simulations, we need to understand the systematic uncertainties associated with the Geant4 Monte-Carlo simulation. We use two different methods to explore these uncertainties: the constant signal efficiency method described below and using alternative Geant 4 hadronic models in the production of proton Monte-Carlo simulations. It should be noted that uncertainty in the energy measurement is not included in the systematic uncertainties. Uncertainty in the energy measurement is still under development and is being estimated in a method similar to the one described in [6]. The energy uncertainty is expected to be comparable to other systematic uncertainties described below and will therefore dominate this analysis and spectral fit to follow.

\subsection{Constant Signal Efficiency}

The constant signal efficiency method tests the stability of the measured cosmic-ray proton spectrum, checking the reproducibility of the measured spectrum for subsets with different acceptances, event rates, and contaminations. The path length of events through the LAT is used to produce energy dependent quantiles.

The path length is used for a number of reasons. Firstly, the path length probes the shower development through interactions with different amounts of active material. As more material is traversed, the energy resolution of the LAT for protons should increase, since more of the EM component of the hadronic shower is contained within the CAL. Secondly, path length probes Geant4's simulation of hadronic shower development in the CAL and how it varies through different amounts of material. These quantiles describe how well the LAT is modeled by looking at these different path cross-sections through the LAT. This analysis uses seven energy dependent quantiles ranging from $90 \%$ to $30 \%$, removing lower path length events with each cut.

For each quantile, we compute an event rate from flight data, acceptance, contamination, and a response matrix using the same methods as described in section 3.2. Once the spectra for each constant signal efficiency is unfolded, the maximum variation for each energy bin is found and set as the error from the constant signal efficiency. Statistical and other systematic errors are not considered in this measurement.

\subsection{Alternative Geant4 Models}

The response matrix (used in unfolding the true energy spectrum) and effective area are all determined from Geant 4 proton simulations. In order to test the quality of our Geant 4 simulations, alternative hadronic models are used for the generation of protons, characterized by different 
shower development, primary interactions, cascade and spallation, and therefore will produce alternative response matrices and effective areas. The different response matrices and effective areas are used in the unfolding of the flight data. The alternative hadronic models alter the energy deposition, and therefore are able to probe the systematic uncertainties associated with the energy estimation.

The available number of hadronic models used in testing is limited due to the version of Geant 4 used in the Pass 8 Event simulation and reconstruction package and degeneracy of hadronic models. Pass 8 uses Geant4 9.4.p04 and, of the available hadronic models, only QBBC and QGS_BIC demonstrate enough differences from the standard list of QGSP_BERT_EPAX to be of interest for this study [12, 13, 14, 15, 16, 17].

Using these alternate instrument response functions, the counts spectrum in true energy is unfolded from the same flight data with the same settings and procedures as with the standard hadronic model described in section 3.2. The maximum variation of each alternate spectra is used to set limits on systematic uncertainty for Geant 4 .

\section{Results and Discussion}

We use 7 years of Pass 8 flight data from August 4, 2008 to July 30, 2015 from $20 \mathrm{GeV}$ to $35 \mathrm{TeV}$ in reconstructed energy. The true energy event rate is unfolded from $54 \mathrm{GeV}$ to $9.5 \mathrm{TeV}$ using the process established in section 3. We add all of the statistical and systematic uncertainties in quadrature and find the differential energy flux shown in Figure 4. The red markers show the statistical uncertainties, the red shaded region shows the statistical and systematic uncertainties without energy uncertainty.

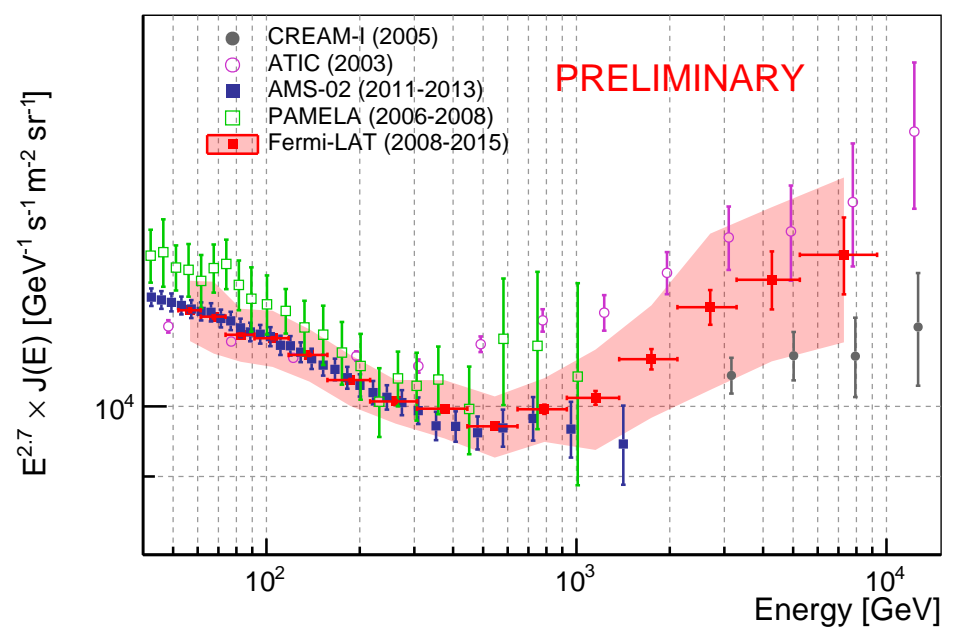

Figure 4: The measured proton spectrum from the Fermi LAT in units of $\mathrm{GeV}^{-1} \mathrm{~s}^{-1} \mathrm{~m}^{-2} \mathrm{sr}^{-1}$ multiplied by $\mathrm{E}^{2.7}$ using 7 years of flight data. The red markers show the statistical uncertainty. The red shaded region shows the sum of the statistical uncertainty, the constant signal efficiency uncertainty 4.1 , and the Geant4 uncertainty 4.2. Uncertainty associated with energy measurement is not included in the plot. Other measurements, which include systematic uncertainties, from PAMELA, AMS-02, ATIC, and CREAM-1, are shown for comparison [1, 2, 3, 4]. 


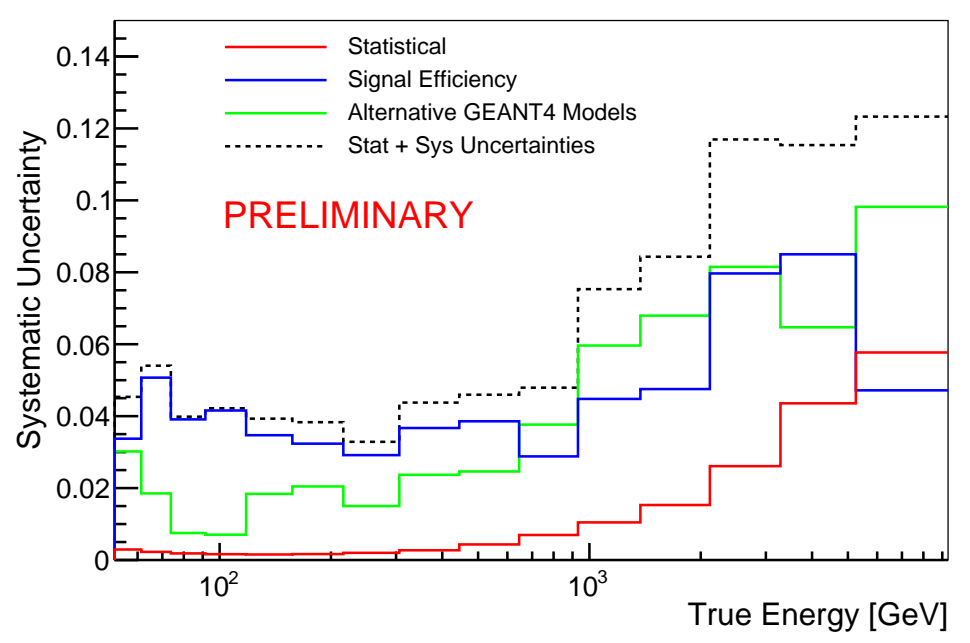

Figure 5: The fractional value of the different contributions to the systematic uncertainties as a function of energy. Blue lines show the uncertainty from the signal efficiency described in section 4.1. Green lines show the uncertainty from Geant4 described in section 4.2. Red lines show the statistical uncertainty. The black dotted line is sum in quadrature of all systematic uncertainty and statistical uncertainty.

The energy dependence of individual components of the systematic uncertainties is shown in Figure 5. The constant signal efficiency uncertainty, which traces the systematic uncertainty associated with the acceptance, is at 5\% across the entire energy range. The Geant 4 uncertainty, which traces the systematic uncertainty associated with the energy estimation, is the largest source of systematic uncertainty at around $10 \%$ across most energies.

This measurement represents the first time a space-based measurement of the cosmic-ray proton spectrum has been able to extend to nearly $10 \mathrm{TeV}$ and observe an energy range covered by both space-based and balloon-borne cosmic-ray experiments. The measured cosmic-ray proton spectrum overlaps with other measurements from PAMELA and AMS-02, extends past the maximum energy measurement of $A M S$ - 02 and additionally overlaps with ATIC and CREAM- 1 measurements $[1,2,3,4]$. The measurement is systematics dominated and is limited by the size of the CAL and its ability to contain and measure hadronic showers, and by the systematic uncertainties associated with Geant4.

\section{Acknowledgments}

The Fermi-LAT Collaboration acknowledges support for LAT development, operation and data analysis from NASA and DOE (United States), CEA/Irfu and IN2P3/CNRS (France), ASI and INFN (Italy), MEXT, KEK, and JAXA (Japan), and the K.A. Wallenberg Foundation, the Swedish Research Council and the National Space Board (Sweden). Science analysis support in the operations phase from INAF (Italy) and CNES (France) is also gratefully acknowledged. 


\section{References}

[1] O. Adriani et al., Pamela measurements of cosmic-ray proton and helium spectra, Science 332 (2011), no. 6025 69-72, [http://science.sciencemag.org/content/332/6025/69.full.pdf].

[2] AMS Collaboration, M. Aguilar et al., Precision measurement of the proton flux in primary cosmic rays from rigidity $1 \mathrm{gv}$ to $1.8 \mathrm{tv}$ with the alpha magnetic spectrometer on the international space station, Phys. Rev. Lett. 114 (Apr, 2015) 171103.

[3] A. D. Panov et al., Energy spectra of abundant nuclei of primary cosmic rays from the data of atic-2 experiment: Final results, Bulletin of the Russian Academy of Sciences: Physics 73 (2009), no. 5 $564-567$.

[4] Y. S. Yoon et al., Cosmic-ray proton and helium spectra from the first cream flight, The Astrophysical Journal 728 (2011), no. 2122.

[5] Fermi-LAT Collaboration, W. B. Atwood, A. A. Abdo, M. Ackermann, W. Althouse, B. Anderson, M. Axelsson, L. Baldini, J. Ballet, D. L. Band, G. Barbiellini, and et al., The Large Area Telescope on the Fermi Gamma-Ray Space Telescope Mission, The Astrophysical Journal 697 (June, 2009) 1071-1102, [arXiv:0902.1089].

[6] Fermi-LAT Collaboration, S. Abdollahi et al., Cosmic-ray electron-positron spectrum from 7 GeV to 2 TeV with the Fermi Large Area Telescope, Phys Rev. D 95 (Apr., 2017) 082007.

[7] S. Schmitt, TUnfold: an algorithm for correcting migration effects in high energy physics, JINST 7 (2012) T10003, [arXiv:1205.6201].

[8] A. N. Tikhonov and V. Y. Arsenin, Solutions of Ill-posed problems. W.H. Winston, 1977.

[9] GEANT4 Collaboration, S. Agostinelli et al., GEANT4: A Simulation toolkit, Nucl. Instrum. Meth. A506 (2003) 250-303.

[10] Fermi-LAT Collaboration, P. Bruel et al., Gamma rays, electrons and positrons up to 3 TeV with the Fermi Gamma-ray Space Telescope, Journal of Physics Conference Series 404 (Dec., 2012) 012033, [arXiv:1210.2558].

[11] R. Brun and F. Rademakers, Root - an object oriented data analysis framework, in AIHENP'96 Workshop, Lausane, vol. 389, pp. 81-86, 1996.

[12] Geant4 Hadronic Working Group Collaboration, V. V. Uzhinsky, The Fritiof (FTF) Model in Geant4, in Proceedings, International Conference on Calorimetry for the High Energy Frontier (CHEF 2013): Paris, France, April 22-25, 2013, pp. 260-264, 2013.

[13] G. Folger and J. P. Wellisch, String parton models in GEANT4, eConf C0303241 (2003) MOMT007, [nucl-th/0306007].

[14] G. Folger, V. N. Ivanchenko, and J. P. Wellisch, The Binary Cascade, European Physical Journal A 21 (Sept., 2004) 407-417.

[15] A. Heikkinen, N. Stepanov, and J. P. Wellisch, Bertini intranuclear cascade implementation in GEANT4, eConf C0303241 (2003) MOMT008, [nucl-th/ 030600 8].

[16] M. Kosov and D. Savin, New exclusive CHIPS-TPT algorithms for simulation of neutron-nuclear reactions, J. Phys. Conf. Ser. 608 (2015), no. 1012050.

[17] Geant4 Hadronic Group Collaboration, J. Yarba, Recent developments and validation of Geant4 hadronic physics, J. Phys. Conf. Ser. 396 (2012) 022060. 